\title{
A Case Report of a Patient with Severe Knee Instability, Treated with Hinge Knee Prosthesis
}

\section{Fezollari L*, Caushi Gj, Asoc Ruci V, Selmani E, Cami E, Gega A, Biba G, Vellku F, Sejdini K, Memini S and Bassi G}

Department of Orthopedics, University Hospital of Trauma, Tirana, Albania

*Corresponding Author: Fezollari L, Department of Orthopedics, University

Hospital of Trauma, Tirana, Albania.
Received: February 26, 2021

Published: July 28, 2021

(C) All rights are reserved by Fezollari L., et al.

\begin{abstract}
A patient who is 59 years old, female with marked knee instability by 8 months after the onset of knee trauma. The patient is unable to walk normally, walking only with knee support. After a careful clinical and radiological examination special knee prosthesis (hinge prosthesis) was recommended.

Keywords: Severe Knee Instability; Hinge Knee Prosthesis; Flexion Movement
\end{abstract}

\section{Introduction and Case Report}

A patient who is 59 years old, female with marked knee instability by 8 months after the onset of knee trauma. The patient is unable to walk normally, walking only with knee support. After a careful clinical and radiological examination special knee prosthesis (hinge prosthesis) was recommended. Hinged prostheses are a solution in cases of severe instability, but their disadvantage is that they have a higher transmission of stress at the bone-implant interface and a pattern of non-physiological movement.

The femoral and tibial nails are needed to tolerate this heavy load and prevent early implant failure. Furthermore, the loaded occupied by the hinge knee prosthesis needs bone resections greater than those made in standard knee prosthesis.

The advantages of hinged knee prostheses is that combines extension and flexion movement with rotation, improving movement mechanics and reducing load transfer with fixation.

\section{Indications}

Indications for the use of a hinge prosthesis are:

- Severe ligamentous laxity with large extension gaps or flexion;
- Large bone destruction in tibial plateau or femoral condyles, with loss of collateral or ACL/PCL ligaments;

- Total rupture of one of the collateral ligaments.

Surgical technique

We used a standard medial parapatellar arthrotomy. Before cementation, abundant lavage was done, and drying with gauze. We didn't touch the patella. Patient was operated under general anesthesia.

A prophylactic antibiotic $2 \mathrm{~g}$ Ceftriaxone was intravenously/bolus administered $30 \mathrm{~min}$ before surgery. Enoxaparin $0.4 \mathrm{ml} \mathrm{sc}$ for 4 weeks postoperatively was administrated.

We used tourniquet in our patient. We kept hemovac for $48 \mathrm{~h}$ postoperatively. According to hemogram analysis blood transfusions was given.

The operation time was 122 minutes. We begin to our patient physiotherapy on the first postoperative day with passive and active ROM. By using crutches we permitted full weight-bearing from the first postoperative day. We recommended maximally for 6 weeks using crutches, after that walking freely without any support. 


\section{Results}

Implanted hinge knee prtosthesis was stable and functional at the time of follow-up with it all components

In follow-up, the patient knee range of motion (ROM) was full extension and flexion at least $80^{\circ}$.

Patient didn't developed any complication (Pulmonary emboli, periprosthetic infection, fracture during surgery, synovitis, dislocation of the prosthesis, fracture after surgery or patellar maltracking, loosening of implants).

\section{X-ray pre-op}
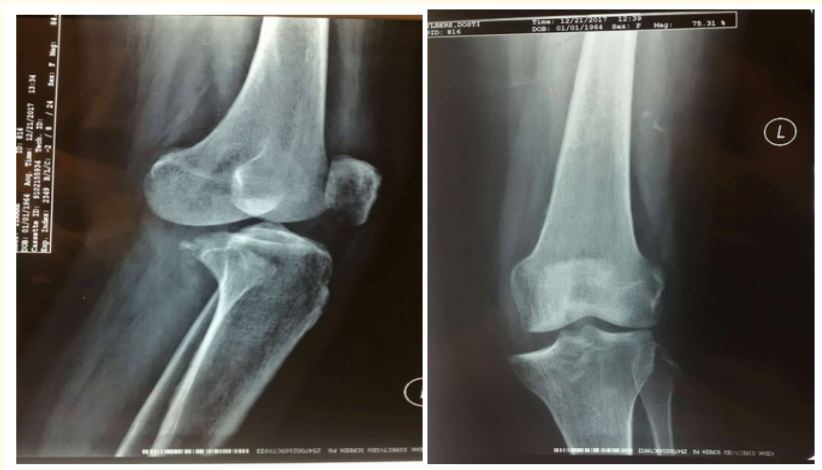

Figure 1

\section{X-ray post-op}
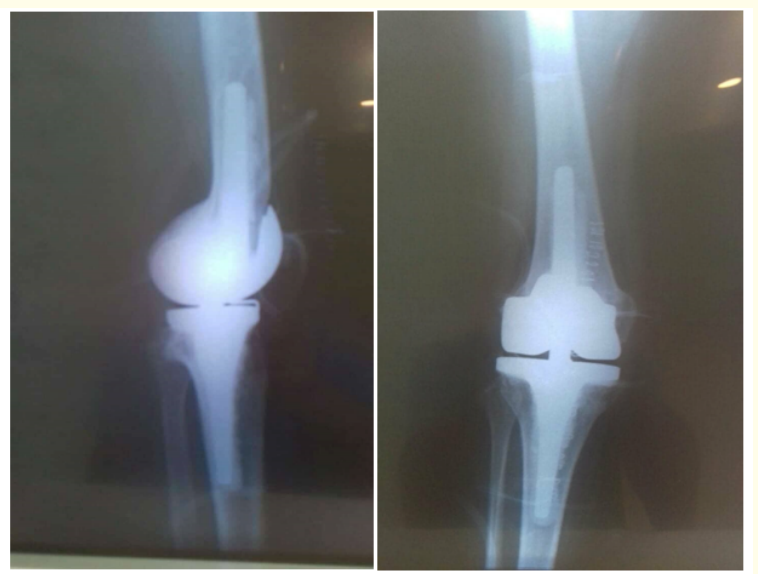

Figure 2

\section{Discussion}

The use of constrained hinge implants in TKA should be reserved for

- Severe ligamentous laxity with large extension gaps or flexion;

- Large bone destruction in tibial plateau or femoral condyles, with loss of collateral or ACL/PCL ligaments;

- Total rupture of one of the collateral ligaments.

However, this type of implant was a good option in upper written indications. The hinge knee prosthesis is used because it decreases the stress on the fixation and allows a more physiological movement of the knee.

Survivorship in a recent review of the literature was in the range of $51 \%$ to $92.5 \%$ at ten years post-operatively. Most ordinary complications rates are in the range of $9.2 \%$ to $63 \%$, with infection and aseptic loosening. Hinge knee prostheses are used in severe knee instability, aseptic loosening, bone loss. They have good survivorship and outcome, but still have high complication and revision conditions. The implant is a good alternative when used properly for patients who are not candidates for less constrained implants [1-9].

\section{Conclusion}

Benefits of Rotating Hinged knee prosthesis was good pain relief, functional recovery and knee stability both in complex primary and in revision cases.

\section{Bibliography}

1. Jamsen E., et al. "Primary knee replacement for primary osteoarthritis in the aged: gender differences in epidemiology and preoperative clinical state". Aging Clinical and Experimental Research 24.6 (2012): 691-698.

2. Nelson CL., et al. "Implant selection in revision total knee arthroplasty". The Journal of Bone and Joint Surgery American 85 (2003): S43-51.

3. Kurtz SM., et al. "Prosthetic joint infection risk after TKA in the Medicare population". Clinical Orthopaedics and Related Research 468.1 (2010): 52-56.

4. Pour AE., et al. "Rotating hinged total knee replacement: use with caution". The Journal of Bone and Joint Surgery American 89.8 (2007): 1735-1741. 
5. Springer BD., et al. "The kinematic rotating hinge prosthesis for complex knee arthroplasty". Clinical Orthopaedics and Related Research 392 (2001): 283-291.

6. Barrack RL. "Evolution of the rotating hinge for complex total knee arthroplasty". Clinical Orthopaedics and Related Research 392 (2001): 292-979.

7. Gehrke T., et al. "The role of hinges in primary total knee replacement". The Bone and Joint Journal 96.11 (2014): 93-95.

8. Zahar A., et al. "Can good infection control be obtained in onestage exchange of the infected TKA to a rotating hinge design? 10-Year results". Clinical Orthopaedics and Related Research 474.1 (2016): 81-87.

9. Angelini FJ., et al. "Knee arthroplasty revision with a constrained implant using hinge and rotating tibial basis". Acta Ortopedica Brasileira 24.1 (2016): 22.

Volume 4 Issue 8 August 2021

(C) All rights are reserved by Fezollari ., et al. 